\title{
HTT2 promotes plant thermotolerance in Brassica rapa
}

Jianxia Jiang ${ }^{1,2}$, Jinjuan Bai ${ }^{1,4}$, Shuxia $\mathrm{Li}^{1,3}$, Xiaorong $\mathrm{Li}^{1,4}$, Liyong Yang ${ }^{2^{*}}$ and Yuke He $\mathrm{C}^{1,4^{*}}$

\begin{abstract}
Background: Numerous regulatory genes participate in plant thermotolerance. In Arabidopsis, HEAT-INDUCED TAS1 TARGET2 (HTT2) is an important thermotolerance gene that is silenced by ta-siR255, a trans-acting siRNA. ta-siR255 is absent from heading Chinese cabbage (Brassica rapa ssp. pekinensis). Our previous attempt to overexpress the endogenous BrpHTT2 gene of heading Chinese cabbage (B. rapa ssp. pekinensis) failed because of cosuppression. In theory, heading Chinese cabbage can overexpress Arabidopsis HTT2 to improve thermotolerance in the absence of ta-siR255-mediated gene silencing and the weak potential of coexpression.

Results: To test the potential application of HTT2 in improving crop thermotolerance, we transferred p35S.:HTT2 to heading Chinese cabbage. We tested the leaf electrical conductivity, hypocotyl elongation, and survival percentage of p35S::HTT2 plants subjected to high-temperature $\left(38^{\circ} \mathrm{C}\right)$ and heat-shock $\left(46^{\circ} \mathrm{C}\right)$ treatment. The leaf electrical conductivity of $p 355$ :.:HTT2 seedlings under high temperature decreased but did negligibly change under heat shock. The hypocotyl length of p355:.:HTT2 seedlings increased under high temperature and heat shock. The survival rate of $p 355$ :.:HTT2 seedlings increased under heat shock. BrpHsfs, a subset of heat-shock factor genes, were upregulated in p35s::HTT2 plants under high-temperature and heat shock conditions. In the field, transgenic plants with HTT2 appeared greener and formed leafy heads earlier than wild-type plants.

Conclusions: Exogenous HTT2 increased the survival rates of heat-shocked heading Chinese cabbage by promoting thermotolerance through decreasing electrical conductivity and extending hypocotyl length. Our work provides a new approach to the genetic manipulation of thermotolerance in crops through the introduction of exogenous thermotolerance genes.
\end{abstract}

Keywords: Brassica rapa, Chinese cabbage, HTT2, Hsf, Thermotolerance

\section{Background}

Crop growth and yield are seriously affected by abiotic stresses, such as heat, cold, drought, waterlogging, and salinity. Among these stresses, high-temperature stress associated with global warming is one of the major threats that may result in extensive losses in global agriculture. Thus, in recent years, numerous researchers have attempted to improve the thermotolerance of crops to decrease yield losses and to ensure global food security.

\footnotetext{
* Correspondence: yangliyong@saas.sh.cn; ykhe@sibs.ac.cn

${ }^{2}$ Crop Breeding and Cultivation Research Institute, Shanghai Academy of Agricultural Sciences, Shanghai 201403, China

${ }^{1}$ National Key Laboratory of Plant Molecular Genetics, Shanghai Institute of Plant Physiology and Ecology, Chinese Academy of Sciences, Shanghai 200032, China

Full list of author information is available at the end of the article
}

Chinese cabbage (Brassica rapa) is one of the most high-yielding and widely planted vegetable crop. The leaves of $B$. rapa supply vital mineral nutrients, crude fiber, and vitamins. However, B. rapa is extremely sensitive to heat stress, especially during its reproductive stage. In recent years, drastic increases in temperature have greatly influenced the yield and quality of $B$. rapa. Therefore, increasing the heat resistance of $B$. rapa is vital for agriculture production.

Trans-acting small interfering RNAs (ta-siRNAs), a unique class of small RNAs, have been recently identified in various plant species. These ta-siRNAs are involved in posttranscriptional gene silencing and are processed as follows: First, functional miRNAs directly bind to noncoding TAS, the precursor RNA of ta-siRNA. The cleavage of TAS is triggered by the binding of miRNAs. Subsequently, double-stranded RNAs (dsRNA) are 
produced from the 3 fragment of TAS through the action of RNA DEPENDENT RNA POLYMERASE6 (RDR6). Then, dsRNAs are processed into phased 21-nt ta-siRNAs by the DCL4 enzyme. SUPPRESSOR OF GENE SILENCING3 interacts and colocalizes with RDR6 in cytoplasmic granules to stabilize the cleaved transcript. Finally, novel ta-siRNAs are produced under the action of nonhomologous miRNAs [1-4]. In Arabidopsis, TAS1, TAS2, TAS3, and TAS4 are four families of noncoding precursor genes that generate ta-siRNAs. Members of the TAS1 family contain three loci, namely TAS1a, TAS1b, and TAS1c, and require miR173 to guide transcript cleavage [5]. After the cleavage of miR173, the TAS1 transcript can form siR480(+)/siR255, siR396(+), and siR438(+).

The expression of some TAS1-derived siRNAs, such as siR480(+)/siR255, is responsive to various abiotic stresses. For example, these siRNAs are down-regulated under salt, dehydration, or cold stress [6, 7]. They target HTT1 (At4g29770), HTT2 (At5g18040), HTT3 (At5g18065), HTT4 (At2g29760), and HTT5 (At1g51670) [2, 6, 8, 9]. Furthermore, the overexpression of HTT2 could enhance the oxidative stress tolerance of transgenic plants [10]. Heat induces the expression of all five target genes of TAS1 siR480(+)/siR255. Microarray and quantitative real-time polymerase chain reaction (qRT-PCR) analyses have demonstrated that heat remarkably induces the expression of HTT1 and HTT2. The overexpression of TAS1 $a$ attenuates thermotolerance by increasing the accumulation of TAS1-siRNAs and decreasing the expression levels of $H T T$ genes. By contrast, the overexpression of rHTT1 or rHTT2 enhances heat resistance. Moreover, several heat-stress transcription factors $(H s f)$ genes are upregulated in $r H T T$ - or $r H T T 2$-overexpressing Arabidopsis seedlings. HTT1 mediates thermotolerance pathways through targeting by TAS1a and activation by HsfA1a [9].

We had previously attempted to overexpress the endogenous BrpHTT2 gene of heading Chinese cabbage (B. rapa ssp. pekinensis) to improve the heat resistance of $B$. rapa. However, our attempt to overexpress BrpHTT2 in heading Chinese cabbage failed because of cosuppression. In this study, we transferred an exogenous Arabidopsis HTT2 gene, which is silenced by trans-acting siRNA ta-siR255, to heading Chinese cabbage, which lacks an endogeneous ta-siR255 biogenesis machinery. As a result, the HTT2 gene was overexpressed in B. rapa, and the thermotolerance of transgenic lines increased. Thus, the HTT2 gene of Arabidopsis is ideal for improving the thermotolerance of B. rapa through genetic manipulation.

\section{Methods}

\section{Plant materials and growth conditions}

Seeds of Bre, an inbred line of heading Chinese cabbage, were germinated for 1 day on moisture-absorbent paper in a plant growth chamber at $22{ }^{\circ} \mathrm{C}$ in the dark. To induce reproductive growth to obtain plants for later use in genetic transformation, the germinated seeds were transferred to a $4{ }^{\circ} \mathrm{C}$ chamber for a 25 -day vernalization period. Then, the seedlings were transplanted into nutrient soil and grown in a green house at $22{ }^{\circ} \mathrm{C}$ for approximately 1 month or until the plants bloomed. The flowering plants were then used for genetic transformation.

\section{Gene cloning and genetic transformation}

The full-length coding sequences of HTT2 were cloned from Arabidopsis by using specific primers (Additional file 1: Table S1). Then, rHTT2 (siR255-resistant versions of HTT2) was obtained and introduced into pCAMBIA1301 binary vectors under the control of the CaMV 35S promoter. The p35S::HTT2 vector was transformed into heading Chinese cabbage cv Bre via the vernalization-infiltration method in accordance with previously described methods [11].

The two lines of $p 35 S:: H T T 2$ transgenic plants (namely HTT2 OE) were designated as HTT2-2 and HTT2-4. Positive HTT2 OE transgenic plants were generated for selection by germinating sterilized seeds on $\mathrm{MS}_{0}$ medium containing $25 \mathrm{mg} / \mathrm{L}$ hygromycin and $250 \mathrm{mg} / \mathrm{L}$ carbenicillin. Hygromycin-resistant seedlings were transplanted into nutrient soil under greenhouse conditions and were identified by using a pair of primers specific for the Hygromycin gene sequence of pCAMBIA1301 binary vectors (Additional file 1: Table S1). Homozygous lines were obtained by self-pollinating positive transgenic plants for three generations.

\section{Leaf electrical conductivity measurements}

The seeds of HTT2-2 and HTT2-4 transgenic plants and Bre were sown on nutrient soil. At the end of $\mathrm{Au}-$ gust 2015, the seedlings were transplanted to the field at the Songjiang Farm Station of Shanghai Institute of Plant Physiology and Ecology. Three large and young leaves were collected from three HTT2-2 and HTT2-4 seedlings in the folding stage. Meanwhile, three leaves were collected from three Bre seedlings. Thus, each line had three biological replicates.

Electrical conductivity was measured as follows: First, a hole puncher was used to obtain 60 leaf discs from each leaf. The leaf discs were transferred to glass test tubes filled with $10 \mathrm{~mL}$ of double-distilled water. Each tube contained 20 leaf discs. The tubes were then capped and marked as transgenic or Bre. The number of replicates was also marked on the tubes. Second, the first group of tubes was incubated for $1 \mathrm{~h}$ in a $22{ }^{\circ} \mathrm{C}$ water bath, the second group was incubated for $1 \mathrm{~h}$ in a $38{ }^{\circ} \mathrm{C}$ water bath, and the third group was incubated for $1 \mathrm{~h}$ in a $46{ }^{\circ} \mathrm{C}$ water bath. Then, the tubes were fetched from the water bath, and subjected to vacuum suction 
until the leaf discs had sunk to the bottom of the tubes, and shaken on an oscillator for $1 \mathrm{~h}$. Each temperature had three replicates. The electrical conductivity of the samples was measured and recorded as R1. The tubes were immediately recapped after measurement. Fifth, the tubes were placed for $20 \mathrm{~min}$ in a $100{ }^{\circ} \mathrm{C}$ water bath. The samples were cooled to $20{ }^{\circ} \mathrm{C}$ after removal from the water bath and shaken for $20 \mathrm{~min}$ on an oscillator. Sixth, the electrical conductivity of the samples was measured and recorded as R2. Electrical conductivity was measured in accordance with the manufacturer's instructions (Mettler-Toledo). Relative electrical conductivity was calculated and recorded as R3 by using the following formula: $R 3=(R 1 / R 2) \times 100 \%$. Each sample had three biological replicates. Two-tailed, unpaired $t$-tests were performed for statistical analysis.

\section{Measurement of hypocotyl length}

The seeds of Bre and HTT2-2 transgenic plants were surface-sterilized for $30 \mathrm{~s}$ with $75 \%$ ethanol and then for 8 min with $0.1 \%$ mercury bichloride. The seeds were washed four times with sterile distilled water and sown onto solid MS medium with $1 \%$ sucrose in petri dishes. The petri dishes were sealed with Parafilm and incubated at $22{ }^{\circ} \mathrm{C}$ in the dark. Most of the seeds germinated after 1 day and were transferred to $2 \mathrm{~mL}$ tubes containing $1 \mathrm{~mL}$ of sterile distilled water. Then, the tubes were separately incubated for $1 \mathrm{~h}$ in a water bath at $22{ }^{\circ} \mathrm{C}$, $38{ }^{\circ} \mathrm{C}$, or $46{ }^{\circ} \mathrm{C}$. After heat treatment, the germinated seeds were sown into the bottles containing $\mathrm{MS}_{0}$ medium and cultivated for 5 days in an illumination incubator at $22{ }^{\circ} \mathrm{C}$ in the dark. After 5 days, the hypocotyl lengths of HTT2-2 and Bre were measured. The numbers of germinated HTT2-2 and Bre seeds under each temperature treatment exceeded 20. $t$-tests were performed to test the significance of differences between HTT2-2 and Bre plants.

\section{Treatment of high temperature and heat shock}

The seeds of Bre and HTT2-2 transgenic plants were surface-sterilized and sown on solid MS medium with $1 \%$ sucrose in sterile bottles. Eight seeds of Bre were sown in the left part of the bottle, and eight seeds of HTT2-2 were sown in the right part of the bottle. In total, 24 seeds of Bre and 24 seeds of HTT2-2 were sown in three bottles. The seeds were cultivated at $22{ }^{\circ} \mathrm{C}$ under long-day conditions ( $16 \mathrm{~h}$ light/ $8 \mathrm{~h}$ dark). After 11 days, the seedlings of HTT2-2 and Bre exhibited vigorous growth and two true leaves. One bottle was incubated for $1 \mathrm{~h}$ in a $46{ }^{\circ} \mathrm{C}$ water bath, another was incubated for $2 \mathrm{~h}$ in a $46{ }^{\circ} \mathrm{C}$ water bath, and the third bottle was incubated for $1 \mathrm{~h}$ in a $22{ }^{\circ} \mathrm{C}$ water bath as the control. The phenotypes that reflected the heat resistance of Bre and HTT2-2 seedlings were observed upon the completion of the heat-shock treatment and at 1 day after treatment. The survival rates of HTT2-2 seedlings were quantified 10 days after heat shock.

\section{qRT-PCR}

Total RNA was extracted from heat-treated samples with Trizol reagent (Invitrogen). Total RNA was treated with DNase I (Takara, Japan) to remove residual genomic DNA. A total of $3 \mu \mathrm{g}$ of total RNA was used for first-strand cDNA synthesis for qRT-PCR with PrimeScript $^{\text {tax }}$ II reverse transcriptase (Takara) and oligo (dT) primers in a $20 \mu \mathrm{L}$ reaction volume in accordance with the manufacturer's instructions. The cDNA reaction mixture was diluted four times, and $2.5 \mu \mathrm{L}$ of the diluted cDNA reaction mixture was used as a template in a $20 \mu \mathrm{L}$ reaction volume. qRT-PCR reactions included the following steps. First, the samples were preincubated at $95{ }^{\circ} \mathrm{C}$ for $3 \mathrm{~min}$. Next, the samples were subjected to 45 cycles of denaturation at $95{ }^{\circ} \mathrm{C}$ for $10 \mathrm{~s}$, annealing at $57{ }^{\circ} \mathrm{C}$ for $20 \mathrm{~s}$, and extension at $72{ }^{\circ} \mathrm{C}$ for $20 \mathrm{~s}$. Finally, the samples were subjected to 61 cycles of $65{ }^{\circ} \mathrm{C}$ for 15 s. The reactions were performed in a MyiQ2 qRT-PCR detection system (Bio-Rad, www.bio-rad.com/) using iQ SYBR Green supermix (Bio-Rad). Each experiment was conducted with three biological replicates, and each sample had three technical replicates. The ACTIN2 gene was used as the reference gene, and relative expression levels were quantified through the $2^{-\Delta \Delta C t}$ method [12]. $t$-tests were performed to analyze the significance of differences. All the primers used for qRT-PCR analysis are listed in Additional file 1: Table S1.

\section{Results}

\section{Sequences and expression of HTT genes in Chinese cabbage}

Arabidopsis possesses four families of noncoding precursor genes. These families include TAS1, TAS2, TAS3, and TAS4. Members of the TAS1 family have three loci-TAS1a, $T A S 1 b$, and TAS1c-and require miR173 to guide transcript cleavage in ta-siRNA generation [5]. The TAS1 loci code for multiple ta-siRNAs, including siR255, siR396(+), and siR438(+) $[2,6,8,13]$. siR255 targets HTT1, HTT2, HTT3, HTT4, and HTT5 [9]. B. rapa is closely related to Arabidopsis, and both plants are crucifers. We used the Brassica database (BRAD) website (http://brassicadb.org/ $\mathrm{brad} /$ ) [14] to search for the homologs of MIR173, TAS1, and $H T T$ in B. rapa. We did not identify any homologs of MIR173 and TAS1 in B. rapa (Fig. 1). Moreover, we did not find siR255 from the small-RNA data of $B$. rapa [15]. The absence of BrpMIR173, BrpTAS1, and siR255 from B. rapa indicates that $B$. rapa lacks the siR255 biogenesis system.

We identified five homologs of HTT genes, namely Bra010276, Bra010277, Bra010278, Bra011210, and Bra039897 in B. rapa. We designated Bra010278 and 


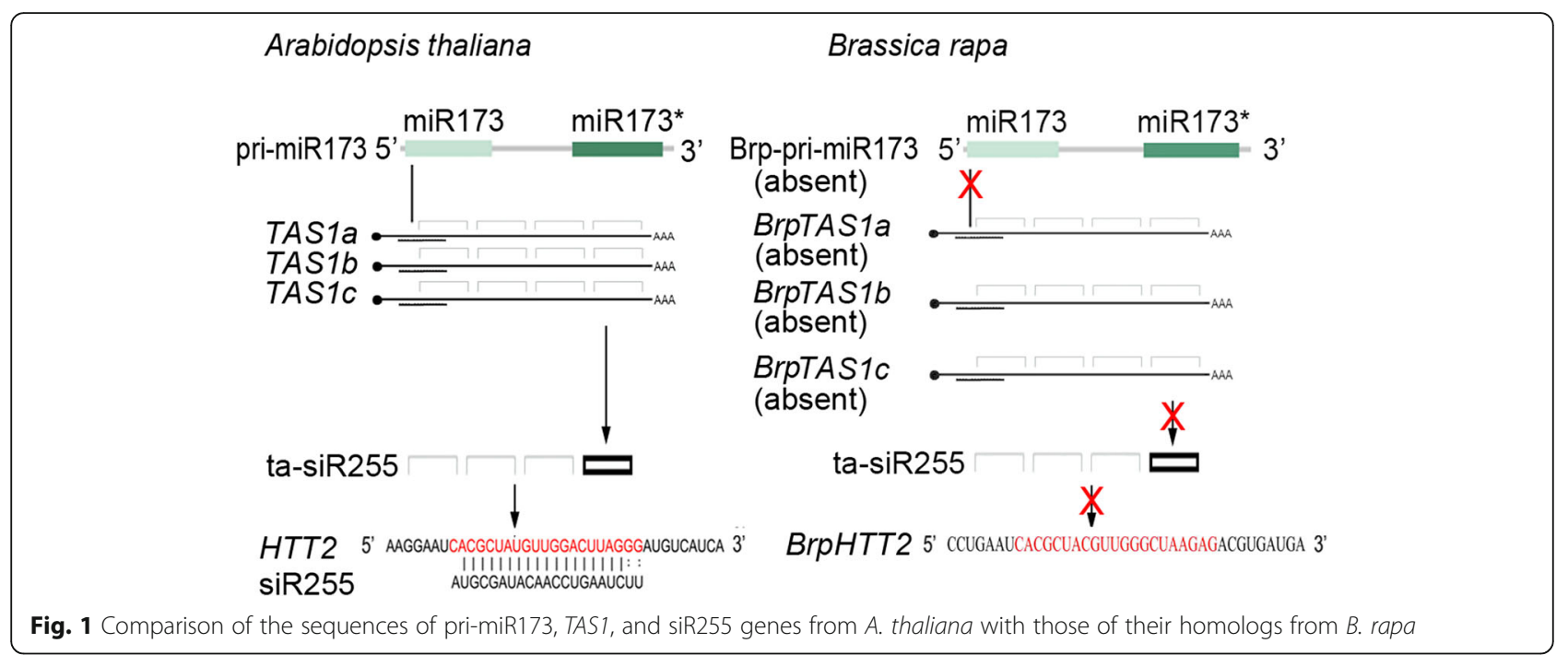

Bra039897 as BrpHTT1a and BrpHTT1b, respectively. We designated Bra011210, Bra010276, and Bra010277 as BrpHTT2, BrpHTT3 and BrpHTT4, respectively. Bra010276, Bra010277, and Bra010278 are three adjacent genes on the A08 chromosome. Bra011210 and Bra039897 are located on the A01 and A07 chromosomes, respectively. The mRNA sequence homology between Bra010276 and Bra010277 is 85.6\%.

HTT1 and HTT2 genes are highly up-regulated in A. thaliana seedlings under heat shock [9]. We performed qRT-PCR to detect the expression levels of BrpHTT2, BrpHTT3, and BrpHTT4 in heat-shocked Bre seedlings. The gene expression levels of BrpHTT2 highly significantly $(p<0.01)$ increased after heat treatment and heat shock. The transcript level of BrpHTT3 slightly increased after heat treatment at $38{ }^{\circ} \mathrm{C}$ and decreased after heat treatment at $46{ }^{\circ} \mathrm{C}$. The expression level of BrpHTT4 remained nearly unchanged after heat treatment (Fig. 2a). These results indicate that similar to that of HTT2 in Arabidopsis, the expression of BrpHTT2 in Bre is induced by heat shock.

\section{$H T T 2$ is overexpressed in $B$. rapa}

We constructed rHTT2 (siR255-resistant versions of Arabidopsis HTT2) (Fig. 2b) under the control of the CAMV 35S promoter [9]. rHTT2 promotes thermotolerance in Arabidopsis. We transferred rHTT2 into Bre via vernalization-infiltration methods [11]. The seeds of $F_{1}$ transgenic plants containing $p 35 S:: r H T T 2$ were obtained through self-pollination, and homologous transgenic lines were selected. The transgenic plants were transferred to the field on August 2017 and 2018. Compared with the seedlings of field-grown wild-type plants, those of transgenic plants appeared greener and formed leafy heads earlier under high-temperature treatment (Fig. 2c). The results of semiquantitative RT-PCR showed that HTT2 is overexpressed in $p 35 S:: r H T T 2$ seedlings (Fig. 2d).

The seedlings of transgenic lines were exposed to heat treatment at $38{ }^{\circ} \mathrm{C}$ or $46{ }^{\circ} \mathrm{C}$ for $1 \mathrm{~h}$. The expression of HTT2 in p35S::rHTT2 seedlings exposed to $38{ }^{\circ} \mathrm{C}$ was up-regulated by $\sim 20$ - and $\sim 5$-fold relative to that in p35S::rHTT2 seedlings exposed to $22{ }^{\circ} \mathrm{C}$ (Fig. 2e) or $46^{\circ} \mathrm{C}$, respectively.

HTT2 decreases the relative leaf electrical conductivity of seedlings subjected to high temperatures and heat shock We conducted the leaf electrical conductivity test to quantify the thermotolerance of $p 35 S:: r H T T 2$ plants. The two transgenic p35S::rHTT2 lines used in this experiment were designated as HTT2-2 and HTT2-4. Leaf discs of equal sizes and numbers were collected from seedlings; immersed in water in test tubes; and incubated for $1 \mathrm{~h}$ in a water bath at $22{ }^{\circ} \mathrm{C}, 38^{\circ} \mathrm{C}$, or $46^{\circ} \mathrm{C}$. The initial electrical conductivity of the leaf discs was measured and recorded as $\mathrm{R} 1$. Then, the leaf discs were incubated in a water bath at $100{ }^{\circ} \mathrm{C}$ for $20 \mathrm{~min}$, and the final electrical conductivity of the leaf discs was measured and recorded as R2. Relative leaf electrical conductivity was calculated and recorded as R3 by using the formula R3 $=(\mathrm{R} 1 / \mathrm{R} 2) \times 100 \%$. Under heat treatment at $38{ }^{\circ} \mathrm{C}$, the relative leaf electrical conductivity values of HTT2-2 and HTT2-4 decreased relative to those of Bre (Fig. 3a, b). Unexpectedly, the relative leaf electrical conductivity values of HTT2-2 and HTT2-4 plants that had been heat shocked at $46{ }^{\circ} \mathrm{C}$ did not decrease. These results indicate that HTT2 decreases electrical conductivity in transgenic plants exposed to $38^{\circ} \mathrm{C}$.

HTT2 increases the hypocotyl length of seedlings exposed to high temperature

We examined the hypocotyl elongation of the transgenic plants. The germinated seeds of HTT2-2 plants were 

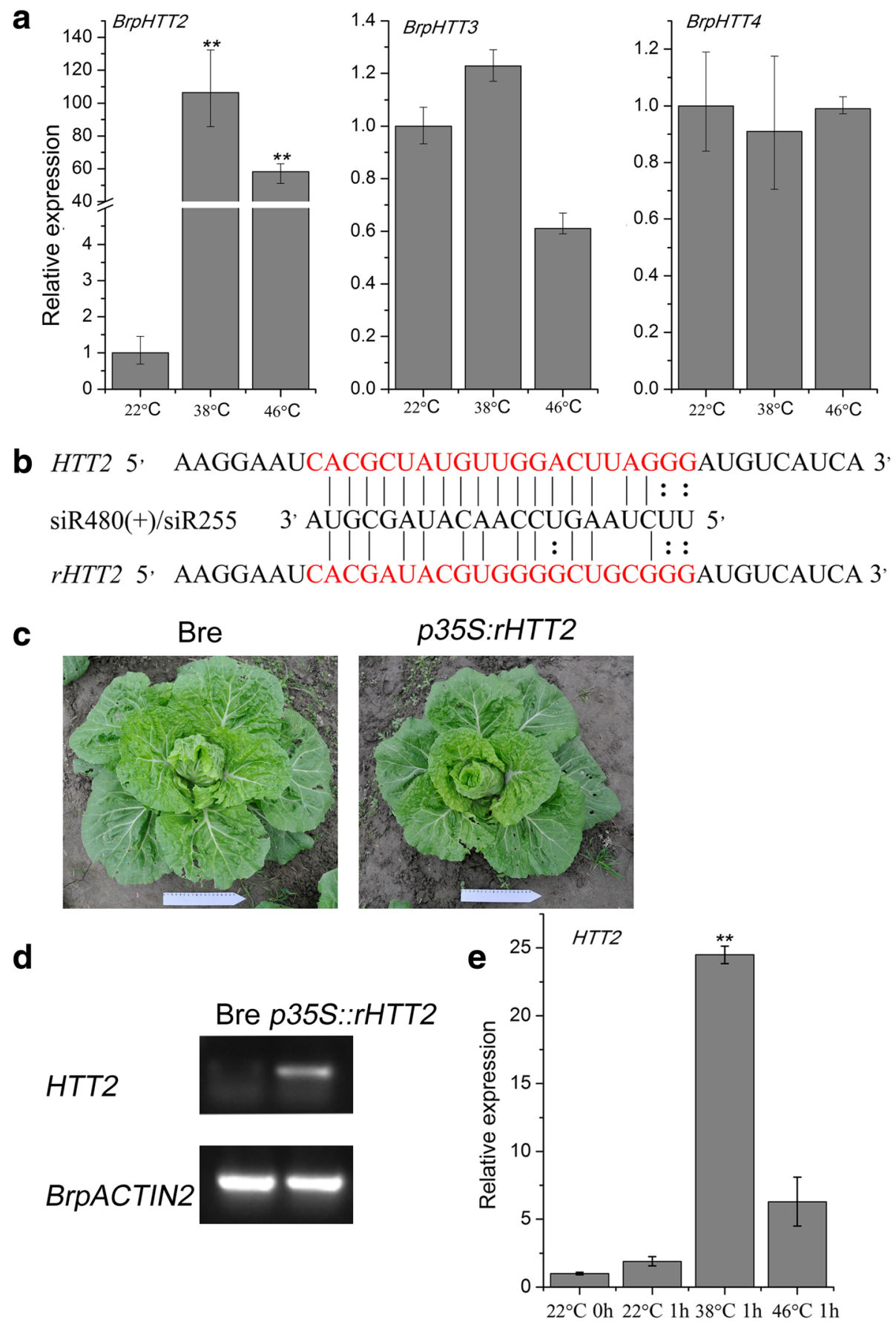

Fig. 2 Expression levels of BrpHTT genes after heat-shock treatment and the phenotype of HTT2-overexpressing transgenic plants. (a) Expression levels of BrpHTT genes after heat-shock treatment. (b) rHTT2 RNA sequences complementary to siR255. (c) HTT2-2 and Bre plants at the folding stage. (d) Quantitative real-time polymerase chain reaction (qRT-PCR) results showing the relative expression levels of HTT2 in Bre and HTT2-2 plants. (e) qRT$P C R$ results showing the relative expression of HTT2 in HTT2-2 plants after heat treatment at different temperatures. Error bars represent standard deviation (SD). Differences were considered significant $\left(^{*}\right)$ if $p<0.05$ (Student's $t$-test) and highly significant $\left(^{* *}\right)$ if $p<0.01$ (Student's t-test).

placed in petri dishes and incubated in a water bath at $22{ }^{\circ} \mathrm{C}, 38{ }^{\circ} \mathrm{C}$, and $46{ }^{\circ} \mathrm{C}$ for $1 \mathrm{~h}$. Then, the germinated seeds were transferred to $\mathrm{MS}_{0}$ medium and grown at $22{ }^{\circ} \mathrm{C}$ in the dark for 5 days. The hypocotyl lengths of the seedlings were measured. After exposure to heat treatment and heat shock, the hypocotyl lengths of HTT2-2 plants were significantly higher than those of wild-type plants (Fig. 3c, d). The suppression of hypocotyl elongation intensified as temperature increased. 

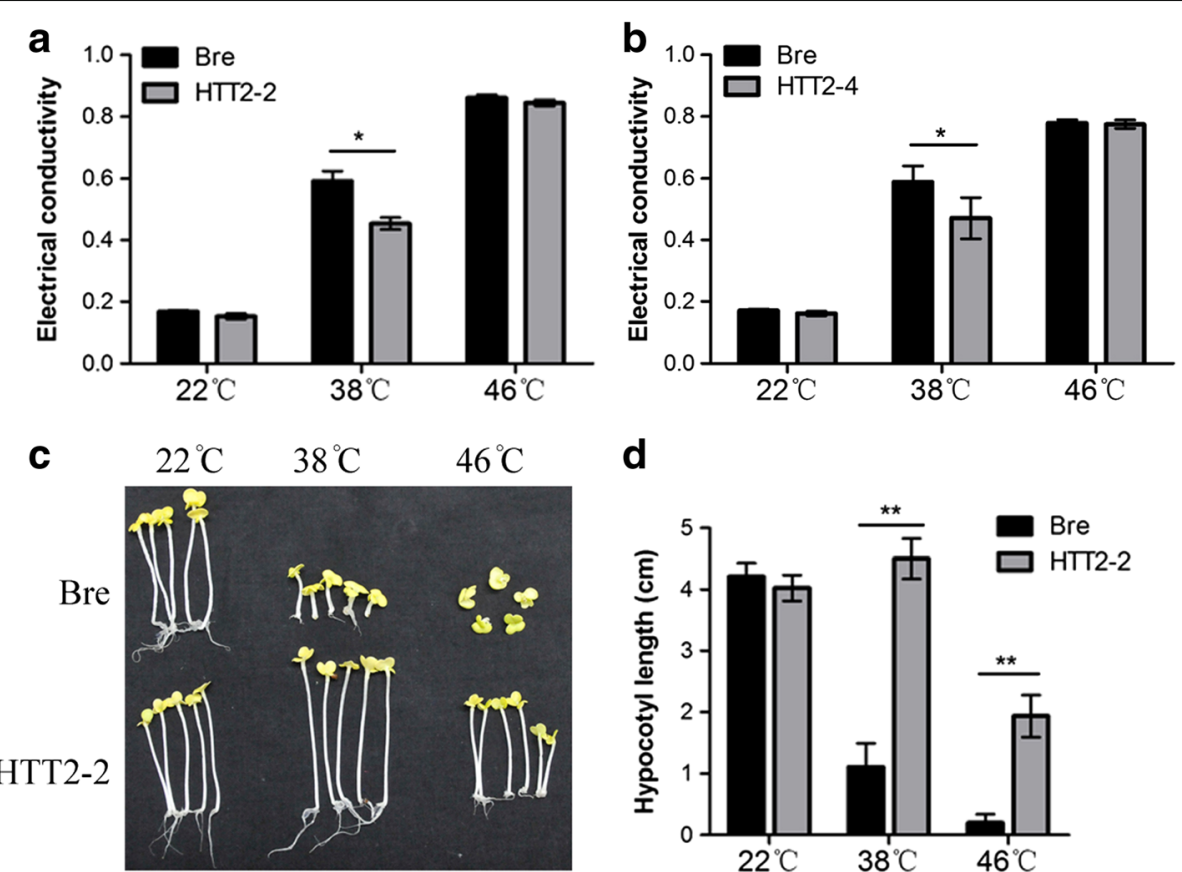

Fig. 3 Electrical conductivity and hypocotyl length of p35S.:rHTT2 plants after high-temperature and heat-shock treatments. (a, b) Leaf electrical conductivity of HTT2-2 (a) and HTT2-4 (b) plants. (c) Hypocotyls of HTT2-2 plants. (d) Hypocotyl length of HTT2-2 plants subjected to heat treatment at different temperatures. Error bars represent SD. More than 20 leaves were measured for each plant of each genotype. The hypocotyl lengths of more than $20 \mathrm{HTT} 2-2$ and Bre seedlings were obtained. Differences between genotypes were considered significant $\left(^{*}\right)$ if $p<0.05$ (Student's $t$-test) and highly significant (**) if $p<0.01$ (Student's $t$-test)

\section{HTT2 enhances thermotolerance after heat shock}

The heat resistance of HTT2-2 seedlings can also be visually observed during heat treatment. Seeds were sown on $\mathrm{MS}_{0}$ medium. After 11 days of incubation at $22{ }^{\circ} \mathrm{C}$ under long-day conditions ( $16 \mathrm{~h}$ light $/ 8 \mathrm{~h}$ dark), the seedlings were incubated in a $46{ }^{\circ} \mathrm{C}$ water bath for 1 and $2 \mathrm{~h}$. After $1 \mathrm{~h}$ of heat-shock treatment at $46{ }^{\circ} \mathrm{C}$, Bre seedlings withered, whereas HTT2-2 seedlings did not (Fig. 4). After $2 \mathrm{~h}$ of heat-shock treatment at $46{ }^{\circ} \mathrm{C}$, Bre seedlings withered severely, whereas HTT2-2 seedlings only withered slightly. Withered Bre seedlings failed to grow and eventually died after being transferred to $22{ }^{\circ} \mathrm{C}$ for further growth. However, the HTT2-2 seedlings that only slightly withered survived, and they continued to grow after they were transferred to $22{ }^{\circ} \mathrm{C}$. After $1 \mathrm{~h}$ of heat-shock treatment at $46{ }^{\circ} \mathrm{C}$, the survival rates of Bre and HTT2-2 seedlings were 57 and $100 \%$, respectively. After $2 \mathrm{~h}$ of heat-shock treatment at $46{ }^{\circ} \mathrm{C}$, the survival

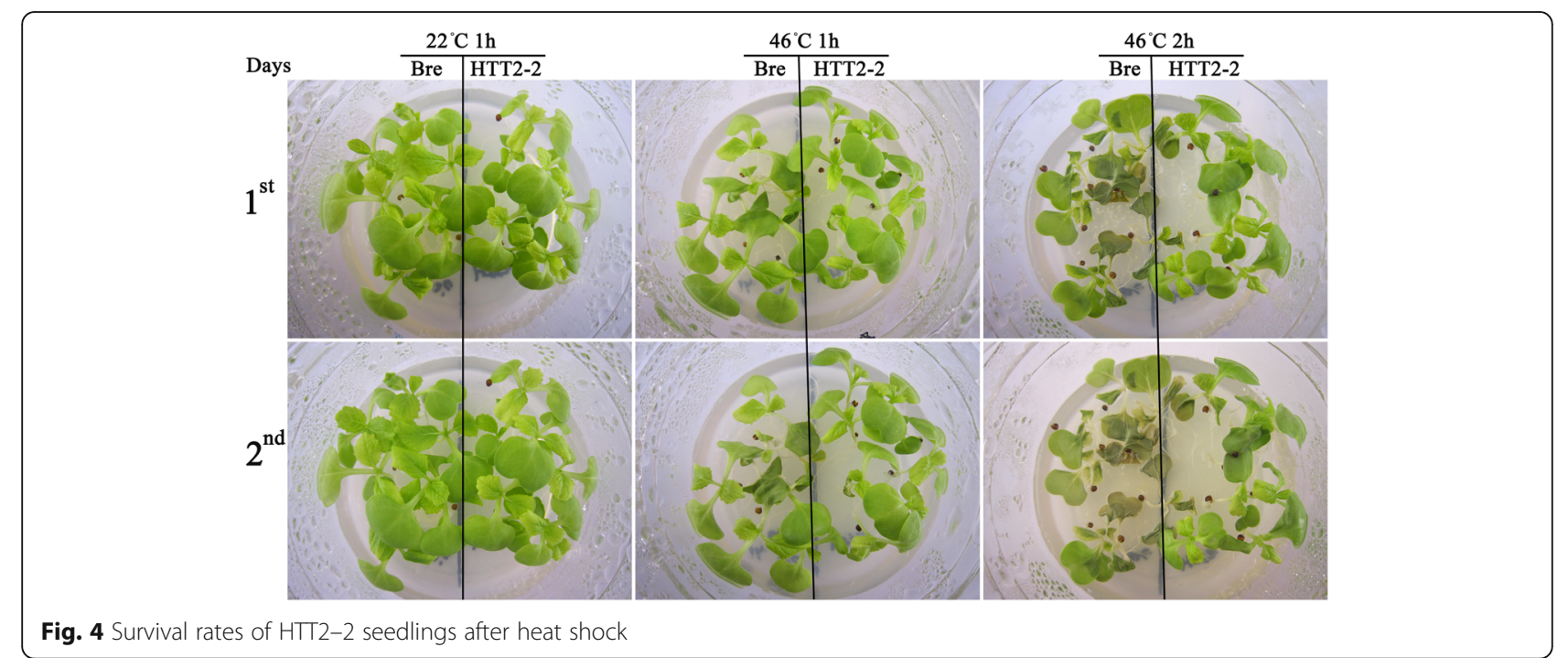


rates of Bre and HTT2-2 seedlings were 0 and $100 \%$, respectively. These results reveal that HTT2 strongly enhances the thermotolerance of HTT2-2 seedlings.

\section{HTT2 up-regulates the expression of some heat-shock factor genes}

Arabidopsis possesses $21 \mathrm{Hsf}$ genes that have important roles in the thermotolerance of plants. Moreover, in Arabidopsis, HTT and Hsf positively regulate each other, and HTT1 mediates thermotolerance via HsfA1a-directed pathways [9]. We selected nine $H s f$ genes from Arabidopsis for further analysis. By searching for the homologous sequences of nine $H s f$ genes in Arabidopsis, we identified 16 homologous genes in B. rapa. We renamed these genes as BrpHsf genes (Table 1).

To explore the roles of $H s f$ genes in heat stress, we selected 14 BrpHsf genes and analyzed their expression levels in the true leaves of HTT2-2 and Bre seedlings that had been subjected for $1 \mathrm{~h}$ to high temperature $\left(38^{\circ} \mathrm{C}\right)$ or heat shock $\left(46{ }^{\circ} \mathrm{C}\right)$. Under normal temperature $\left(22^{\circ} \mathrm{C}\right)$, the expression levels of BrpHsfA1d-1, BrpHsfA3, and BrpHsfA1d-2 were higher in HTT2-2 plants than in wild-type plants while those of BrpHsfA1a, BrpHsfAle-1, BrpHsfA1b-1, BrpHsfAle-2, BrpHsfA1b-2, and BrpHsfAle-3 were downregulated (Fig. 5a). Notably, the expression levels of BrpHsfA7a-1, BrpHsfB2b-2, BrpHsfA7b, BrpHsfA2, and $B r p H s f B 2 b-1$ were significantly up-regulated ( $p$-value $<0.05$ ) in HTT2-2 plants relative to that in wild-type plants after exposure to heat-shock treatment at $38^{\circ} \mathrm{C}$ or $46^{\circ} \mathrm{C}$ (Fig. 5b).

\section{Discussion}

HTT genes are the target genes of TAS1-siRNAs. The expression levels of HTT1 and HTT2 are drastically up-regulated in $A$. thaliana seedlings in response to heat shock, whereas the expression of TAS1a is inhibited by heat [9]. We found that HTT2 gene of Arabidopsis is highly expressed in B. rapa. We used qRT-PCR to analyze the expression levels of BrpHTT2 to BrpHTT4. The over-expression of HTT2 in $B$. rapa may be attributed to the following reasons: First, the TAS1 transcripts and siR255 that act on the posttranscriptional silencing of the HTT2 transcript are absent. Second, HTT2 and BrpHTT do not cosuppress each other given their dissimilar sequences. Instead, the expression level of HTT2 in HTT2-2 plants was up-regulated after heat treatment. In cosuppression, when a gene is introduced into a cell through transformation, neither the resident nor the transgene copy of the same gene is expressed (repeat-induced gene silencing) [16]. Apparently, HTT2 is not cosuppressed by BrpHTT genes. Thus, this gene has potential applications in improving the thermotolerance of Brassica crops.

In HTT2-2 plants, electrical conductivity decreases after high-temperature treatment but not after heat-shock treatment, whereas hypocotyl length increases after high-temperature and heat-shock treatments, compared with those of wild-type. Hypocotyl elongation is a morphological indicator of the thermotolerance of Brassica crops under extremely high temperature. By contrast, electrical conductivity is a physiological indicator of heat response and thus cannot be used as an index of thermotolerance. The survival rates of heat-shocked HTT2-2 plants are considerably higher than those of heat-shocked wild-type plants. These results indicate that the over-expression of HTT2 in $B$. rapa greatly enhances heat resistance.

A total of 21 different $H s f$ genes have been identified in A. thaliana. These genes are assigned to the three major classes $\mathrm{A}, \mathrm{B}$, and $\mathrm{C}$ on the basis of the phylogeny of their DNA binding domains and the organization of their hydrophobic repeats [17]. HsfA1 members, which belong to group 1 in class A and include $H s f A 1 a, H s f A 1 b$, $H s f A 1 d$, and $H s f A 1 e$, are responsive to heat shock [18-21].

Table 1 Hsf genes in Arabidopsis thaliana and their homologs in Brassica rapa

\begin{tabular}{|c|c|c|c|}
\hline Hsf genes in A. thaliana & Homologous genes in B. rapa & & \\
\hline AtHsfA1a (At4g17750) & BrpHsfAla (Bra040179) & & \\
\hline AtHsfA1b (At5g16820) & BrpHsfA1b-1 (Bra008593) & BrpHsfA1b-2 (Bra023584) & \\
\hline AtHsfAld (At1g32330) & $\begin{array}{l}\text { BrpHsfAld-1 } \\
\text { (Bra023258) }\end{array}$ & $\begin{array}{l}\text { BrpHsfAld-2 } \\
\text { (Bra035507) }\end{array}$ & \\
\hline AtHsfAle (At3g02990) & BrpHsfAle-1 (Bra032023) & $\begin{array}{l}\text { BrpHsfAle-2 } \\
\text { (Bra021381) }\end{array}$ & $\begin{array}{l}\text { BrpHsfAle-3 } \\
\text { (Bra001071) }\end{array}$ \\
\hline AtHsfA2 (At2g26150) & BrpHsfA2 (Bra000557) & & \\
\hline AtHsfA3 (At5g03720) & $\begin{array}{l}\text { BrpHsfA3 } \\
\text { (Bra009515) }\end{array}$ & & \\
\hline AtHsfA7a (At3g51910) & $\begin{array}{l}\text { BrpHsfA7a-1 } \\
\text { (Bra012828) }\end{array}$ & $\begin{array}{l}\text { BrpHsfA7a-2 } \\
\text { (Bra012829) }\end{array}$ & $\begin{array}{l}\text { BrpHsfA7a-3 } \\
\text { (Bra033468) }\end{array}$ \\
\hline AtHsfA7b (At3g63350) & $\begin{array}{l}\text { BrpHsfA7b } \\
\text { (Bra007739) }\end{array}$ & & \\
\hline AtHsfB2b (At4g11660) & $\begin{array}{l}\text { BrpHsfB2b-1 } \\
\text { (Bra040968) }\end{array}$ & $\begin{array}{l}\text { BrpHsfB2b-2 } \\
\text { (Bra000749) }\end{array}$ & \\
\hline
\end{tabular}



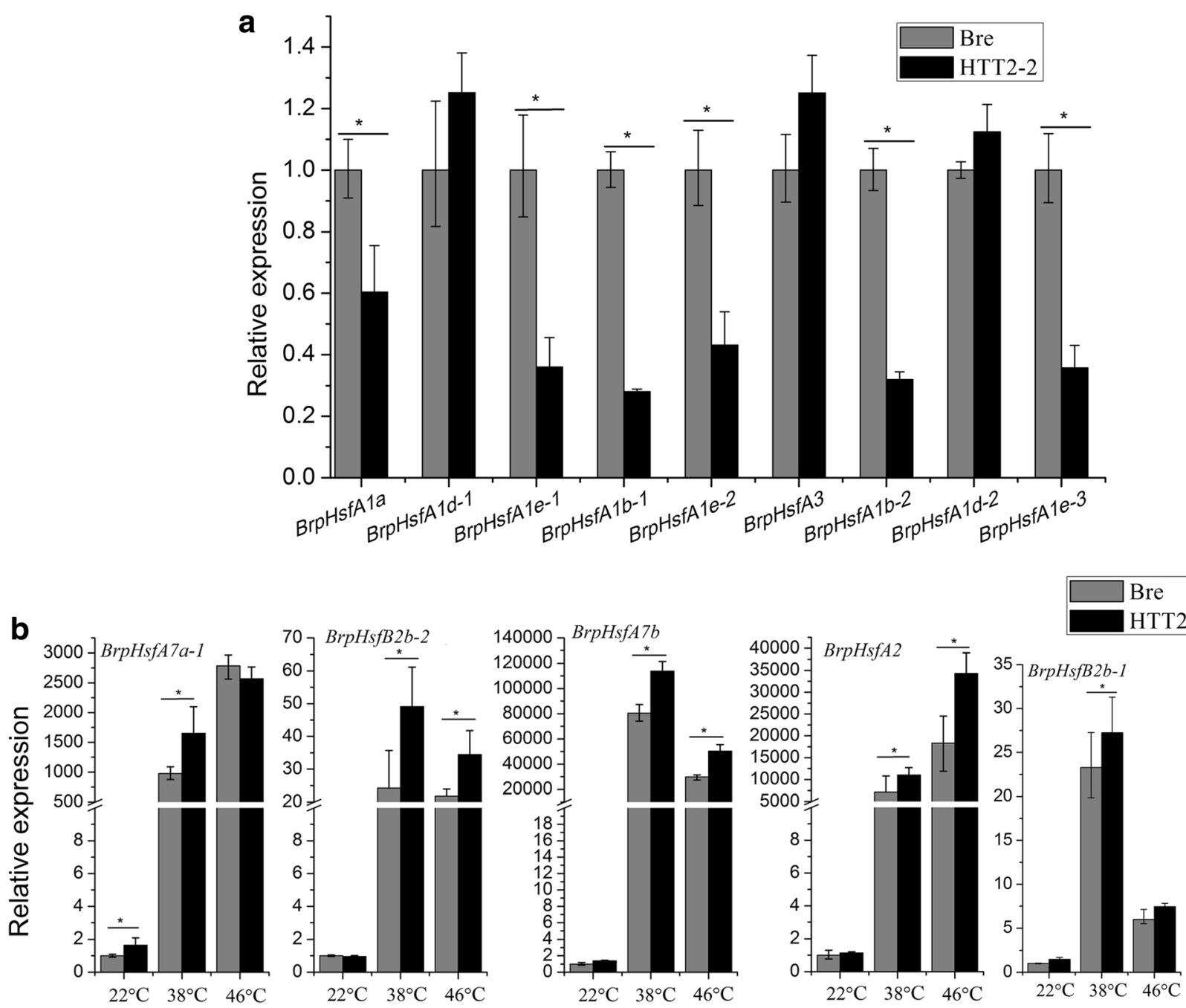

Fig. 5 Effects of HTT2 overexpression on the expression levels of BrpHsf genes. a Expression levels of BrpHsf genes in HTT2-2 seedlings under normal temperature $\left(22^{\circ} \mathrm{C}\right)$. b Expression levels of BrpHsf genes in $\mathrm{HTT} 2-2$ seedlings after heat treatment at different temperatures. Error bars represent SD. The relative expression abundance of each gene was measured with three biological replicates. Differences were considered significant $\left(^{*}\right)$ if $p<0.05$ (Student's $t$-test) and highly significant $\left(^{* *}\right)$ if $p<0.01$ (Student's $t$-test)

HsfAld and HsfAle are involved in the transcriptional regulation of $H s f A 2$ and function as key regulators in the $H s f$ signaling network in response to heat stress and high light [20]. Hsf3 depresses heat-shock response and confers thermotolerance when over-expressed in transgenic plants [22]. Li et al. [9] found a regulatory relationship between $H T T$ and Hsf genes in Arabidopsis. Qian et al. [23] identified a regulatory relationship between HsfAla and Hsp genes. We detected the expression levels of 14 BrpHsf genes in Bre and HTT2-2 after high-temperature $\left(38{ }^{\circ} \mathrm{C}\right)$ and heat-shock $\left(46{ }^{\circ} \mathrm{C}\right)$ treatments. We found that the expression levels of BrpHsfA1d-1, BrpHsfA3, and BrpHsfA1d-2 are higher in HTT2-2 plants than in wild-type plants under normal temperature conditions $\left(22{ }^{\circ} \mathrm{C}\right)$. Notably, the up-regulation of BrpHsfA7a-1, BrpHsfB2b-2, BrpHsfA7b,
BrpHsfA2, and BrpHsfB2b-1 expression after either high-temperature or heat-shock treatment is more pronounced in HTT2-2 plants than in wild-type plants. The HTT2 gene up-regulates the expression of several $B r p H s f$ genes, especially under high or extremely high temperatures. We conclude that HTT2 gene promotes plant thermotolerance by activating crucial heat-shock factor genes.

\section{Conclusions}

Our results reveal that exogenous HTT2 increases the survival rate of $B$. rapa seedlings under heat-shock treatment by promoting thermotolerance through reducing electrical conductivity and increasing hypocotyl length. Our work provides a new approach to the genetic manipulation of crop thermotolerance through the introduction of exogenous thermotolerance genes. 


\section{Additional file}

Additional file 1: Table S1. Primers for expression analysis of qRT-PCR, CDNA cloning of HTT2, and identification of HPT gene in transgenic plants. (DOCX $20 \mathrm{~kb})$

\section{Abbreviations}

Hsf: heat-stress transcription factors; HTT2: HEAT-INDUCED TAS1 TARGET2; qRTPCR: quantitative real-time polymerase chain reaction; RDR6: RNADEPENDENT RNA POLYMERASE6; TAS: precursor RNA of ta-siRNA; tasiRNA: transacting small interfering RNAs

\section{Acknowledgements}

The authors would like to thank Mr. Pinglin Liu for growing and observation of plants in the field.

\section{Funding}

This work was supported by grants from the National Key Research and Development Program of China (Grant No.2016YFD0101900), Natural Science Foundation of China (Grant No. 31471883 and 31571261), and Shanghai Agriculture Applied Technology Development Program, China (Grant No. Z20160109).

\section{Availability of data and materials}

The datasets generated and analyzed during the current study are available in the NCBI Banklt repository, under the ID 2119196.

\section{Authors' contributions}

$\mathrm{YH}$ conceived the project and research plan and finished the manuscript. JJ, BJ and SL performed the experiments and drafted the manuscript. XL analyzed the data. LY co-supervised and complemented the writing. All authors have read and approved the final manuscript.

\section{Ethics approval and consent to participate}

Not applicable.

\section{Consent for publication}

Not applicable.

\section{Competing interests}

The authors declare that they have no competing interests.

\section{Publisher's Note}

Springer Nature remains neutral with regard to jurisdictional claims in published maps and institutional affiliations.

\section{Author details \\ ${ }^{1}$ National Key Laboratory of Plant Molecular Genetics, Shanghai Institute of Plant Physiology and Ecology, Chinese Academy of Sciences, Shanghai 200032, China. ${ }^{2}$ Crop Breeding and Cultivation Research Institute, Shanghai Academy of Agricultural Sciences, Shanghai 201403, China. ${ }^{3}$ Institute of Tropical Bioscience and Biotechnology, Chinese Academy of Tropical Agricultural Sciences, Haikou 571101, China. ${ }^{4}$ CAS Center for Excellence in Molecular Plant Sciences, Institute of Plant Physiology and Ecology, Chinese Academy of Sciences, Shanghai, China.}

\section{Received: 3 April 2018 Accepted: 7 June 2018}

Published online: 20 June 2018

\section{References}

1. Peragine A, Yoshikawa M, Wu G, Albrecht HL, Poethig RS. SGS3 and SGS2/ SDE1/RDR6 are required for juvenile development and the production of trans-acting siRNAs in Arabidopsis. Genes Dev. 2004;18(19):2368-79.

2. Yoshikawa M, Peragine A, Park MY, Poethig RS. A pathway for the biogenesis of trans-acting siRNAs in Arabidopsis. Genes Dev. 2005;19(18): 2164-75.

3. Xie Z, Allen E, Wilken A, Carrington JC. DICER-LIKE 4 functions in transacting small interfering RNA biogenesis and vegetative phase change in Arabidopsis thaliana. P Natl Acad Sci USA. 2005;102(36):12984-9.
4. Vaucheret $\mathrm{H}$. MicroRNA-dependent trans-acting siRNA production. Sci STKE. 2005; 2005(300):pe43.

5. Allen E, Xie Z, Gustafson AM, Carrington JC. microRNA-directed phasing during trans-acting siRNA biogenesis in plants. Cell. 2005;121(2):207-21.

6. Sunkar R, Zhu JK. Novel and stress-regulated microRNAs and other small RNAs from Arabidopsis. The Plant Cell Online. 2004;16(8):2001-19.

7. Kume K, Tsutsumi K, Saitoh Y. TAS1 trans-acting siRNA targets are differentially regulated at low temperature, and TAS1 trans-acting siRNA mediates temperature-controlled At1g51670 expression. Biosci Biotechnol Biochem. 2010;74(7):1435-40

8. Vazquez F, Vaucheret H, Rajagopalan R, Lepers C, Gasciolli V, Mallory AC, Hilbert JL, Bartel DP, Crété P. Endogenous trans-acting siRNAs regulate the accumulation of Arabidopsis mRNAs. Mol Cell. 2004;16(1):69-79.

9. Li SX, LiU JX, LiU ZY, Li XR, WU FJ, He YK. HEAT-INDUCED TAS1 TARGET1mediates thermotolerance via HEAT STRESS TRANSCRIPTION FACTOR A1a-directed pathways in Arabidopsis. Plant Cell. 2014;26(4):1764-80.

10. Luhua S, Ciftci-Yilmaz S, Harper J, Cushman J, Mittler R. Enhanced tolerance to oxidative stress in transgenic Arabidopsis plants expressing proteins of unknown function. Plant Physiol. 2008;148(1):280-92.

11. Bai JJ, Wu FJ, Mao YF, He YK. In planta transformation of Brassica rapa and B. napus via vernalization-infiltration methods. Protocol Exchange. 2013. https://doi.org/10.1038/protex.2013.067.

12. Livak KJ, Schmittgen TD. Analysis of relative gene expression data using real-time quantitative PCR and the $2^{-\Delta \Delta C T}$ method. Methods. 2001;25(4): 402-8.

13. Park W, Li J, Song R, Messing J, Chen X. CARPEL FACTORY, a Dicer homolog, and HEN1, a novel protein, act in microRNA metabolism in Arabidopsis thaliana. Curr Biol. 2002;12:1484-95.

14. Cheng F, Liu SY, Wu J, Fang L, Sun SL, Liu B, Li PX, Hua W, Wang XW. BRAD, the genetics and genomics database for Brassica plants. BMC Plant Biol. 2011;11:136-41.

15. Yu X, Wang H, Lu Y, de Ruiter M, Cariaso M, Prins M, van Tunen A, He Y. Identification of conserved and novel microRNAs that are responsive to heat stress in Brassica rapa. J Exp Bot. 2012;63:1025-38.

16. Jorgensen RA. Cosuppression, flower color patterns, and metastable gene expression states. Science. 1995;268:686-91.

17. Nover L, Bharti K, Doring P, Mishra SK, Ganguli A, Scharf KD. Arabidopsis and the heat stress transcription factor world: how many heat stress transcription factors do we need? Cell Stress Chaperones. 2001;6(3):177-89.

18. Lohmann C, Eggers-Schumacher G, Wunderlich M, Schoffl F. Two different heat shock transcription factors regulate immediate early expression of stress genes in Arabidopsis. Mol Gen Genomics. 2004;271(1):11-21.

19. Liu HC, Liao HT, Charng YY. The role of class A1 heat shock factors (HSFA1s) in response to heat and other stresses in Arabidopsis. Plant Cell Environ. 2011;34(5):738-51

20. Nishizawa-Yokoi A, Nosaka R, Hayashi H, Tainaka H, Maruta T, Tamoi M, Ikeda M, Ohme-Takagi M, Yoshimura K, Yabuta Y, et al. HsfA1d and HsfAle involved in the transcriptional regulation of HsfA2 function as key regulators for the Hsf signaling network in response to environmental stress. Plant Cell Physiol. 2011:52(5):933-45.

21. Yoshida T, Ohama N, Nakajima J, Kidokoro S, Mizoi J, Nakashima K, Maruyama K, Kim JM, Seki M, Todaka D, et al. Arabidopsis HsfA1 transcription factors function as the main positive regulators in heat shockresponsive gene expression. Mol Gen Genomics. 2011;286(5-6):321-32.

22. Prändl $R$, Hinderhofer $K$, Eggers-Schumacher $G$, Schöffl F. HSF3, a new heat shock factor from Arabidopsis thaliana, derepresses the heat shock response and confers thermotolerance when overexpressed in transgenic plants. Mol Gen Genet. 1998;258(3):269-78.

23. Qian J, Chen J, Liu YF, Yang LL, Li WP, Zhang LM. Overexpression of Arabidopsis HsfA1a enhances diverse stress tolerance by promoting stressinduced Hsp expression. Genet Mol Res. 2014;13(1):1233-43. 